\title{
Matching and stabilization of discrete mechanical systems
}

\author{
Anthony M. Bloch ${ }^{1}$, Melvin Leok ${ }^{2}$, Jerrold E. Marsden ${ }^{3}$, and Dmitry V. Zenkov ${ }^{4}$ * \\ ${ }^{1}$ Department of Mathematics, University of Michigan, Ann Arbor, MI 48109, USA. \\ 2 Department of Mathematics, Purdue University, West Lafayette, IN 47907, USA. \\ ${ }^{3}$ Control and Dynamical Systems, California Institute of Technology 107-8, Pasadena, CA 91125, USA. \\ ${ }^{4}$ Department of Mathematics, North Carolina State University, Raleigh, NC 27695, USA.
}

\begin{abstract}
Controlled Lagrangian and matching techniques are developed for the stabilization of equilibria of discrete mechanical systems with symmetry as well as broken symmetry. Interesting new phenomena arise in the controlled Lagrangian approach in the discrete context that are not present in the continuous theory. Specifically, a nonconservative force that is necessary for matching in the discrete setting is introduced. The paper also discusses digital and model predictive controllers.
\end{abstract}

(C) 2007 WILEY-VCH Verlag GmbH \& Co. KGaA, Weinheim

\section{Introduction}

In the controlled Lagrangian approach, one considers a mechanical system with kinetic energy invariant with respect to a Lie group action on the configuration space. To stabilize an equilibrium, the kinetic and potential energies are modified to produce a controlled Lagrangian. The equations corresponding to this controlled Lagrangian are the closed-loop equations and the new terms appearing in those equations correspond to control inputs. The modifications to the Lagrangian are chosen so that no new terms appear in the equations for the variables that are not directly controlled. One advantage of this approach is that once the form of the control law is derived using the controlled Lagrangian, the stability of an equilibrium of the closed-loop system can be determined by energy methods, using any available freedom in the choice of the parameters of the controlled Lagrangian. To obtain asymptotic stabilization, dissipation-emulating terms are added to the control input.

The main objective of this paper is to develop the method of controlled Lagrangians for discrete mechanical systems. As the closed loop dynamics of a controlled Lagrangian system is itself Lagrangian, it is natural to adopt a variational discretization that exhibits good long-time numerical stability (see [5]). This study is also motivated by the recent development of structurepreserving algorithms for the numerical simulation of discrete controlled systems (eg., discrete optimization).

The matching procedure is carried out explicitly for discrete systems with one shape and one group degree of freedom to avoid technical issues and to concentrate on the new phenomena that emerge in the discrete setting that have not been observed in the continuous-time theory. It is also shown that once spectrally stabilized, the equilibria of interest can be asymptotically stabilized by adding dissipation-emulating terms. The discrete controlled dynamics is used to construct a real-time model predictive controller with piecewise constant control inputs. This serves to illustrate how discrete mechanics can be naturally applied to yield digital controllers for mechanical systems.

\section{Discrete Matching}

For simplicity, we consider systems with one shape and one group degree of freedom and assume that the configuration space $Q$ is the direct product of a one-dimensional shape space $S$ and a one-dimensional Lie group $G$. The points in $Q$ are written as $q=(\phi, s)$, with $\phi \in S$ and $s \in G$. In the rest of the paper, we will adopt the notations $q_{k}=\left(\phi_{k}, s_{k}\right) \in Q$, $q_{k+1 / 2}=\left(q_{k}+q_{k+1}\right) / 2$, and $\Delta q_{k}=q_{k+1}-q_{k}$. The discretization is done in the spirit of discrete variational mechanics, as in [5]. Given a continuous-time Lagrangian $L(q, \dot{q})=\frac{1}{2}\left[\alpha \dot{\phi}^{2}+2 \beta(\phi) \dot{\phi} \dot{s}+\gamma \dot{s}^{2}\right]-V_{1}(\phi)-V_{2}(s)^{1}$ we define a second-order accurate discrete Lagrangian by the formula $L^{d}\left(q_{k}, q_{k+1}\right)=h L\left(q_{k+1 / 2}, \Delta q_{k} / h\right)$, where $h>0$ is the time step. The discrete dynamics is governed by the discrete Euler-Lagrange equations

$$
\partial_{\phi_{k}} L^{d}\left(q_{k}, q_{k+1}\right)+\partial_{\phi_{k}} L^{d}\left(q_{k-1}, q_{k}\right)=0, \quad \partial_{s_{k}} L^{d}\left(q_{k}, q_{k+1}\right)+\partial_{s_{k}} L^{d}\left(q_{k-1}, q_{k}\right)=-u_{k},
$$

where $u_{k}$ is the control input. The shape variable is not directly actuated.

The kinetic energy is assumed to be $G$-invariant, but the potential energy is not necessarily $G$-invariant. The objective is to feedback stabilize an equilibrium $(\phi, s)=\left(\phi_{e}, s_{e}\right) .^{2}$ Following [2], the continuous-time controlled Lagrangian is defined by

$$
L_{\tau, \sigma, \rho, \epsilon}(\phi, s, \dot{\phi}, \dot{s})=L(\phi, s, \dot{\phi}, \dot{s}+\tau(\phi) \dot{\phi})+\frac{1}{2} \sigma \gamma(\tau(\phi) \dot{\phi})^{2}+\frac{1}{2}(\rho-1) \gamma(\dot{s}-(\sigma-1) \tau(\phi) \dot{\phi})^{2}+V_{2}(s)-V_{\epsilon}(y),
$$

* Corresponding author E-mail: dvzenkov@ncsu.edu, Phone: +19195154201, Fax: +1 9195137336

1 See [2] for the motivation of the structure of the potential energy.

2 In the absence of control, system (1) may fail to have this equilibrium. 
where $\sigma$ and $\rho$ are constants, $\tau(\phi)=-\beta(\phi) / \sigma \rho, y=s-(1 / \sigma+1 / \rho-1) / \gamma \int_{\phi_{e}}^{\phi} \beta(z) d z$, and $V_{\epsilon}(y)$ is an arbitrary negativedefinite function.

Motivated by the continuous-time matching procedure (see [2]), we define the discrete controlled Lagrangian by the formula $L_{\tau, \sigma, \rho, \epsilon}^{d}\left(q_{k}, q_{k+1}\right)=h L_{\tau, \sigma, \rho, \epsilon}\left(q_{k+1 / 2}, \Delta q_{k} / h\right)$. The dynamics associated with this Lagrangian is amended by the term $w_{k}$ in the discrete shape equation:

$$
\partial_{\phi_{k}} L_{\tau, \sigma, \rho, \epsilon}^{d}\left(q_{k}, q_{k+1}\right)+\partial_{\phi_{k}} L_{\tau, \sigma, \rho, \epsilon}^{d}\left(q_{k-1}, q_{k}\right)=-w_{k}, \quad \partial_{s_{k}} L_{\tau, \sigma, \rho, \epsilon}^{d}\left(q_{k}, q_{k+1}\right)+\partial_{s_{k}} L_{\tau, \sigma, \rho, \epsilon}^{d}\left(q_{k-1}, q_{k}\right)=0
$$

This term $w_{k}$ is important for matching systems (1) and (2). The presence of the term $w_{k}$ represents an interesting (but manageable) departure from the continuous theory.

The discrete controlled momentum is defined by the formula $J_{k}=\rho \gamma\left(\Delta s_{k} / h-(\sigma-1) \tau\left(\phi_{k+\frac{1}{2}}\right) \Delta \phi_{k} / h\right)$. The following statement is proved by a straightforward calculation:

Theorem 2.1 The dynamics (1) and (2) are the same if and only if $u_{k}$ and $w_{k}$ are given by

$$
\begin{aligned}
u_{k}=\frac{h}{2}\left[V_{2}^{\prime}\left(s_{k+\frac{1}{2}}\right)+\right. & \left.V_{2}^{\prime}\left(s_{k-\frac{1}{2}}\right)\right]-\frac{h}{2 \rho}\left[V_{\epsilon}^{\prime}\left(s_{k+\frac{1}{2}}\right)+V_{\epsilon}^{\prime}\left(s_{k-\frac{1}{2}}\right)\right]-\frac{\gamma \Delta \phi_{k} \tau\left(\phi_{k+\frac{1}{2}}\right)-\gamma \Delta \phi_{k-1} \tau\left(\phi_{k-\frac{1}{2}}\right)}{h}, \\
w_{k}=\left(1-\sigma+\frac{\sigma}{\rho}\right)( & \tau\left(\phi_{k+\frac{1}{2}}\right)\left[-\gamma \rho J_{k}+\frac{h}{2} V_{\epsilon}^{\prime}\left(y_{k+\frac{1}{2}}\right)\right] \\
& \left.\quad+\tau\left(\phi_{k-\frac{1}{2}}\right)\left[\gamma \rho J_{k-1}+\frac{h}{2} V_{\epsilon}^{\prime}\left(y_{k-\frac{1}{2}}\right)\right]-\tau^{\prime}\left(\phi_{k+\frac{1}{2}}\right) J_{k} \Delta \phi_{k}-\tau^{\prime}\left(\phi_{k-\frac{1}{2}}\right) J_{k-1} \Delta \phi_{k-1}\right) .
\end{aligned}
$$

The stability of equilibria of the controlled system is established by means of an analysis of the spectrum of the linearized discrete equations. The equilibrium to be stabilized is written as $\left(\phi_{k}, s_{k}\right)=\left(\phi_{e}, s_{e}\right)$. Following [2], we modify the control input (3) by adding the discrete dissipation-emulating term $D\left(\Delta y_{k-1}+\Delta y_{k}\right) / 2 h$ in order to achieve the asymptotic stabilization. In the above, $D$ is a constant.

Theorem 2.2 The equilibrium $\left(\phi_{k}, s_{k}\right)=\left(\phi_{e}, s_{e}\right)$ of equations (2) is asymptotically stable if $-\beta^{2}\left(\phi_{e}\right) /\left(\alpha \gamma-\beta^{2}\left(\phi_{e}\right)\right)<$ $\sigma<0, \rho<0, V_{\epsilon}^{\prime \prime}\left(s_{e}\right)<0$, and $D$ is positive.

Remark. If the potential energy of the system is group-invariant ( $s$-independent), one can study a less restrictive problem of orbital stabilization of relative equilibria $\phi_{k}=\phi_{e}, \Delta s_{k}=$ const. In this case the second equation in (2) becomes the discrete controlled momentum conservation law. One can show that dynamics (1) reduced to the momentum level $J_{k}=p$ and dynamics (2) with $w_{k}=0$ reduced to the momentum level $J_{k}=\mu$ are equivalent if $\mu=p /(1-1 / \sigma)$. See [3] and [4] for details.

We now explore the use of the forced discrete Euler-Lagrange equations as the model in a real-time model predictive controller, with piecewise constant control forces. The digital controller uses the position information it senses for $t=$ $-2 h,-h$ to estimate the positions at $t=0, h$ during the time interval $[-h, 0]$. This allows it to compute a symmetric finite difference approximation $u_{1 / 2}=u\left(\left(\bar{\phi}_{0}+\bar{\phi}_{1}\right) / 2,\left(\bar{s}_{0}+\bar{s}_{1}\right) / 2,\left(\bar{\phi}_{1}-\bar{\phi}_{0}\right) / h,\left(\bar{s}_{1}-\bar{s}_{0}\right) / h\right)$ to the continuous control force $u(\phi, s, \dot{\phi}, \dot{s})$ at $t=h / 2$. In the above, the overbar indicates that the position variable is being estimated by the numerical model. This control is then applied as a constant control input for the time interval $[0, h]$. The process is then repeated for the next time interval. Simulations for the benchmark cart-pendulum example demonstrate that the piecewise constant controller introduces dissipation-like effects, which are reduced as the time-step is decreased (see [4] for details).

Acknowledgements The research of AMB was supported by NSF grants DMS-0305837, DMS-0604307, and CMS-0408542. The research of ML was partially supported by NSF grants DMS-0504747 and DMS-0726263, and a University of Michigan Rackham faculty grant. The research of JEM was partially supported by AFOSR Contract FA9550-05-1-0343. The research of DVZ was partially supported by NSF grants DMS-0306017 and DMS-0604108.

\section{References}

[1] Bloch, A. M., N.E. Leonard, \& J.E. Marsden, Controlled Lagrangians and the Stabilization of Mechanical Systems I: The First Matching Theorem, IEEE Trans. on Systems and Control 45, 2253-2270, (2000).

[2] Bloch, A. M., D-E. Chang, N. E. Leonard, \& J. E. Marsden, Controlled Lagrangians and the Stabilization of Mechanical Systems II: Potential Shaping, Trans. IEEE on Autom. Contr. 46, 1556-1571, (2001).

[3] Bloch, A. M., M. Leok, J.E. Marsden, \& D. V. Zenkov, Controlled Lagrangians and Stabilization of the Discrete Cart-Pendulum System, Proc. CDC 44, 6579-6584, (2005).

[4] Bloch, A. M., M. Leok, J.E. Marsden, \& D. V. Zenkov, Controlled Lagrangians and Stabilization of Discrete Mechanical Systems I, (submitted), (2007).

[5] Marsden, J. E. \& M. West, Discrete Mechanics and Variational Integrators, Acta Numerica 10, 357-514, (2001). 